\title{
Investigation into the quantitative and qualitative characteristics of choroidal melanoma through magnetic resonance imaging and B-scan ultrasound
}

This article was published in the following Dove Press journal:

Clinical Ophthalmology

22 August 2017

Number of times this article has been viewed

\author{
Vassilis Papayiannis' \\ Konstantinos T Tsaousis ${ }^{2,3}$ \\ Constantinos A \\ Kouskouras ${ }^{4}$ \\ Afroditi Haritanti ${ }^{4}$ \\ Vasilios F Diakonis ${ }^{5}$ \\ loannis T Tsinopoulos 2 \\ 'Department of Radiology, \\ Papageorgiou General Hospital, \\ 2Department of Ophthalmology, \\ Aristotle University of Thessaloniki, \\ Thessaloniki, Greece; ${ }^{3}$ John A Moran \\ Eye Center, University of Utah, Salt \\ Lake City, UT, USA; ${ }^{4}$ Department of \\ Radiology, AHEPA Aristotle University \\ Hospital of Thessaloniki, Thessaloniki, \\ Greece; ${ }^{5}$ Bascom Palmer Eye Institute, \\ Miller School of Medicine, University \\ of Miami, Miami, FL, USA
}

Correspondence: Konstantinos

T Tsaousis

John A Moran Eye Center, University of Utah, 65 Mario Capecchi Drive, Salt Lake City, UT 84I32, USA

Email konstantinos.tsaousis@gmail.com
Objective: To investigate the homogeneity and vascularity of choroidal melanoma through magnetic resonance imaging (MRI) and brightness modulation (B-mode) ultrasound scan and their correlation with dimensions of tumor, as well as to measure the sensitivity of both modalities in retinal detachment (RD) detection.

Materials and methods: This retrospective chart review included patients diagnosed with choroidal melanoma. All these patients underwent MRI scans using T2-weighted (T2-WI) and T1-weighted (T1-WI) sequences, before and after an intravenous injection of paramagnetic contrast material. The patients were also examined using a B-mode ultrasound scan, and the results from both modalities were compared (tumor homogeneity, tumor height, tumor base diameter, and tumor vascularity).

Results: Forty-two patients (mean age $=65.33 \pm 12.51$ years) with choroidal melanoma were included in the study. Homogeneity was confirmed in 16 patients through ultrasound scan, in 19 patients through T1-WI sequence, in 21 patients through T2-WI sequence, and in 25 patients through T1-WI sequence + contrast (gadolinium). Patients with homogenous tumors presented with lower $(P=0.0045)$ mean height than that of those with nonhomogenous tumors, whereas no statistically significant difference was found for base diameter measurements $(P=0.056)$. Patients with tumors of high vascularity presented with greater mean height $(P=0.000638)$ and greater mean base diameter compared with those with tumors of low vascularity $(P=0.019543)$. RD was detected in 26 patients through T1-WI sequence, in 13 patients through T2-WI sequence, in 26 patients through T1-WI sequence + contrast, and in 32 patients through ultrasound scan, which proved to be the most sensitive modality.

Conclusion: The height of choroidal melanoma was positively correlated with tumor's homogeneity. Melanomas of greater height were found to be less homogenous, due to increased degeneration and higher occurrence of intratumoral hemorrhage. In addition, choroidal melanoma's height was also positively correlated with the level of its vascularity. Finally, ultrasound scan was found to be more sensitive than MRI in the detection of RD.

Keywords: homogeneity, vascularity, ultrasound scan, MRI, imaging characteristics, retinal detachment

\section{Introduction}

Choroidal melanoma represents a principal ocular pathology, and it is the most frequent primary intraophthalmic malignancy that can lead patients to death. It is rather rare in general population, with an incidence of about 5.3-10.9 cases in every 1,000,000 people. ${ }^{1,2}$ Most previously published series report that the diagnosis is usually set during the sixth decade of life, with a mean age of 55 years. ${ }^{3}$ Clinically, choroidal 
melanoma can cause a decrease in visual function, if located at the posterior pole (macula). Peripherally located choroidal melanoma usually presents with a visual field defect or the sense of a focal flickering light that corresponds to the tumor location.

Despite the fact that novel methods have been used in the imaging of choroidal tumors, such as enhanced depth imaging optical coherence tomography (EDI-OCT), ${ }^{4,5}$ swept source optical coherence tomography (SS-OCT), ${ }^{6}$ and even contrast-enhanced ultrasound (CEUS), ${ }^{7}$ the best established imaging modalities for the diagnosis and monitoring of choroidal melanoma remain ultrasound scan and magnetic resonance imaging (MRI). Malignant melanoma's ultrasonographic characteristics are its size, shape, dense composition, low reflectivity, mostly homogenous structure, and vascularization. ${ }^{8}$ Its typical MRI appearance consists of high signal intensity on T1-weighted (T1-WI) sequence and low signal intensity on T2-weighted (T2-WI) sequence, due to the paramagnetic properties of melanin. Mild-to-moderate arterial enhancement after intravenous (IV) bolus administration of paramagnetic contrast agent is also noted. ${ }^{9,10}$ Both vascularity and homogeneity are clinically important for monitoring and prognosis of choroidal melanoma. ${ }^{11,12}$

The aim of the present study was to investigate the baseline characteristics of choroidal melanoma through ultrasound scan and MRI. An investigation in patients with homogenous and high-vascularity tumors was attempted with respect to the tumor size that was determined by height and base diameter measurements. In addition, the sensitivity of ultrasound scan and MRI regarding retinal detachment (RD) was also assessed.

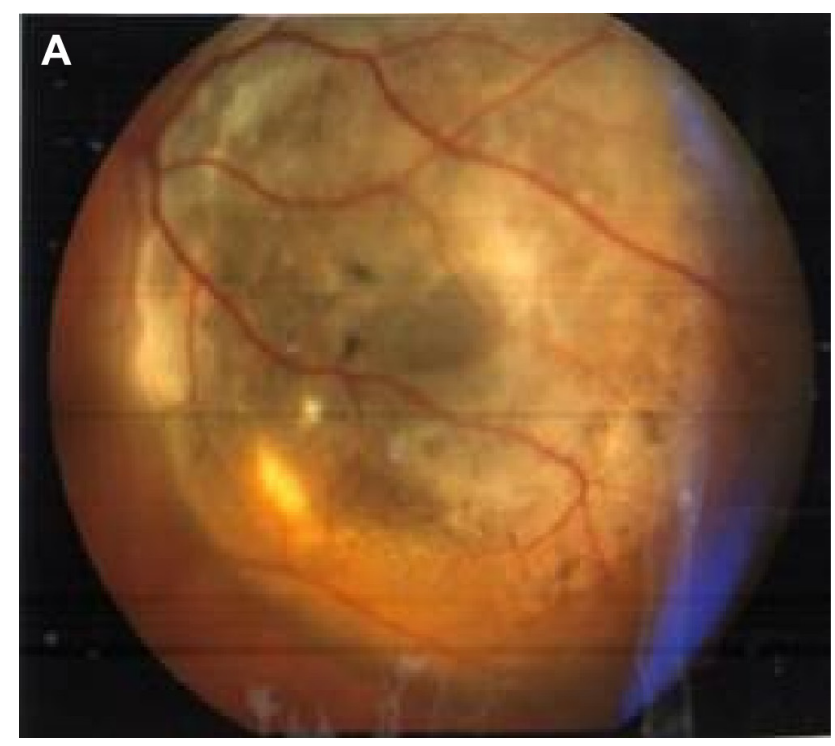

\section{Materials and methods Study setting}

This retrospective study was conducted at Papageorgiou General Hospital and AHEPA Aristotle University Hospital in Thessaloniki, Greece. The study protocol was approved by the Ethics Committee of the Aristotle University of Thessaloniki Medical School. Patients' consent to review their medical records was not required by the institutional review board, as the study does not report on primary research. All the data analyzed were collected as part of routine diagnosis. Examination tests, which were done without fail and as part of routine care, were essential for confirming the diagnosis and for classifying the patients and were in no way an add-on for purpose of research. Data are de-identified; anonymity and confidentiality were guaranteed.

\section{Participants}

A retrospective chart review was performed to identify the patients with choroidal melanoma in two university-based outpatient services (Figure 1A). Inclusion criteria were the diagnosis of choroidal melanoma and the ability to understand the nature and aims of the examinations performed.

\section{Patient assessment}

The ultrasound scanning was conducted by using the highfrequency ultrasounds emitting transducers, working at brightness modulation (B-mode; Siemens Sonoline G60S, L 10-5, Munich, Germany, and Philips iU22 Xmatrix, L 12-5, Amsterdam, the Netherlands). Orbital anatomy was scanned in transverse, longitudinal, and oblique planes, in order to illustrate the ultrasonographic characteristics of choroidal

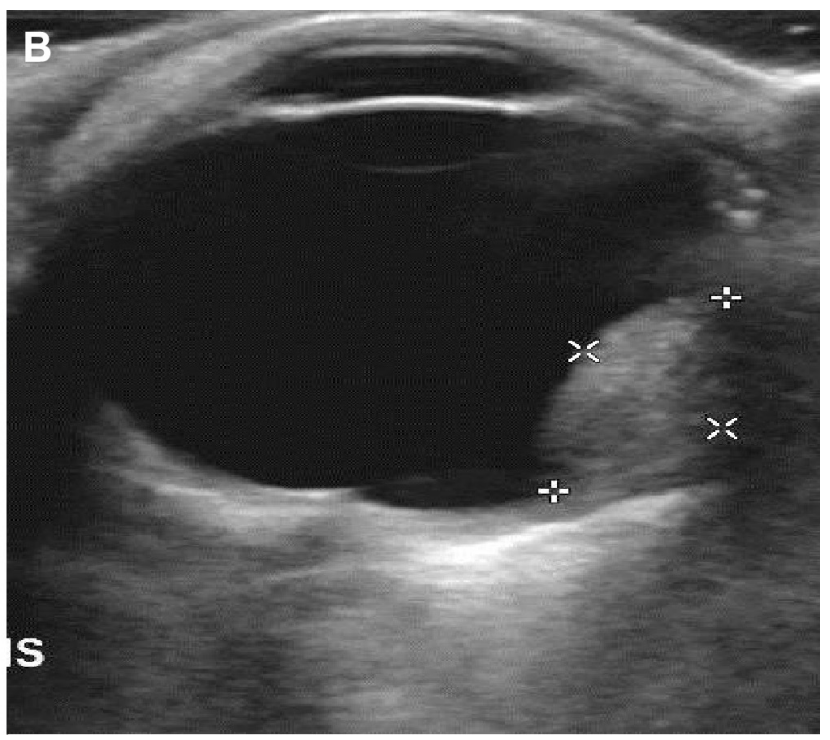

Figure I (A) Fundoscopy image of ocular melanoma; (B) measurements of height and maximum base diameter for choroidal melanoma on ultrasound scan. 
melanoma and measure its size (height and base diameter; Figure 1B). Tumor vascularity, flow range, and velocity were investigated using color Doppler technique, and this technique depicts ultrasound frequency changes when the ultrasound beam is reflected by red blood cells, allowing the evaluation of blood flow velocity. Moreover, power Doppler technique increases flow detection sensitivity for small vessels with low blood flow velocity and therefore is valuable in the evaluation of tumor's neovascularization.

In MRI, the orbits were scanned by using a head coil following a protocol of axial plane sections of 3-mm slice thickness, complemented by at least one more, vertical to the tumor's long axis. Images were obtained at T2-WI and T1-WI sequences before and after an IV injection of paramagnetic contrast material, as well as at fat suppression sequences (Siemens Somatom Plus 1.5 T).

\section{Examined parameters}

Homogeneity as a typical imaging characteristic of choroidal melanoma

When using B-mode ultrasound scan, choroidal melanoma is usually depicted as a principally homogeneous tumor ${ }^{13}$ (Figure 2A), presenting low ultrasound reflectivity, whereas no significant intermediate spikes can be detected when using amplitude modulation ultrasound (A-mode).

\section{Correlation of choroidal melanoma's size with tumor's homogeneity}

On MRI, an even spread of signal intensity throughout the tumor is considered as a typical finding for choroidal melanoma, otherwise referred to as homogenous appearance ${ }^{14}$ (Figure 3A-F). MRI homogeneity findings were shown to vary, according to one of the two basic measurements of choroidal melanoma's size (ie, height or base diameter).
Correlation of choroidal melanoma's size with tumor's vascularity

Vascularity was measured by using ultrasound Doppler techniques that have shown great success for the depiction of neovascularization, vascular architecture, patency, and flow rates (Figure 2B).

\section{Ultrasound scan and MRI sensitivity on RD}

Concerning concurring $\mathrm{RD}$, both of these modalities have been widely used in clinical practice. ${ }^{15}$

\section{Statistical methods}

Data were collected and analyzed using Microsoft Excel 2007 for Windows (Microsoft Corporation, Redmond, WA, USA) and SPSS for Windows, version 16.0 (SPSS Inc., Chicago, IL, USA).

\section{Results}

In total, 42 patients ( 20 women and 22 men) with choroidal melanoma were included in the study. The mean age of the participants was $65.33 \pm 12.51$ years.

\section{Homogeneity}

Homogeneity of choroidal lesions (melanomas) was confirmed in 16 patients through ultrasound scan, in 19 patients through T1-WI sequence, in 21 patients through T2-WI sequence, and in 25 patients through T1-WI sequence + contrast (gadolinium). The results are presented in Figure 4.

\section{Correlation of choroidal melanoma's size with tumor's homogeneity}

We also attempted to compare homogenous tumors with nonhomogenous ones in terms of their height and base diameter measurements. According to the previously mentioned
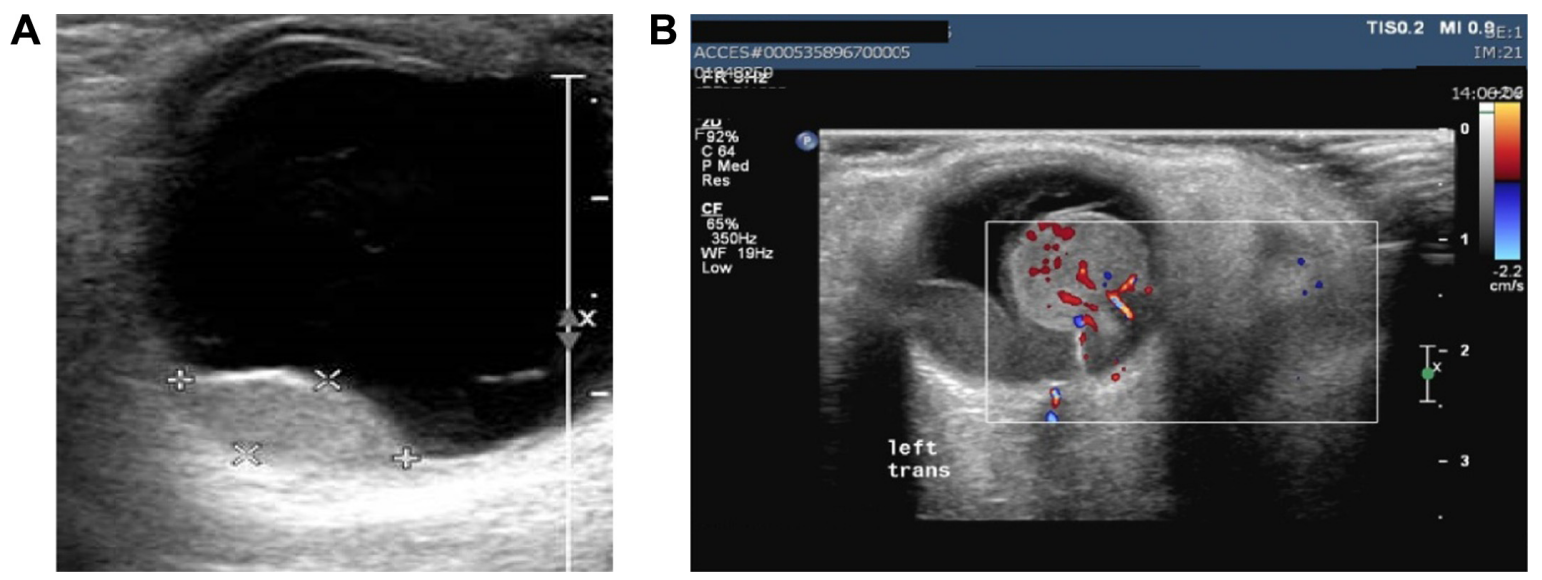

Figure 2 (A) Characteristic homogeneous appearance of choroidal melanoma on ultrasound scans; (B) application of Doppler technique depicting and enabling the study of tumor's neovascularization. 

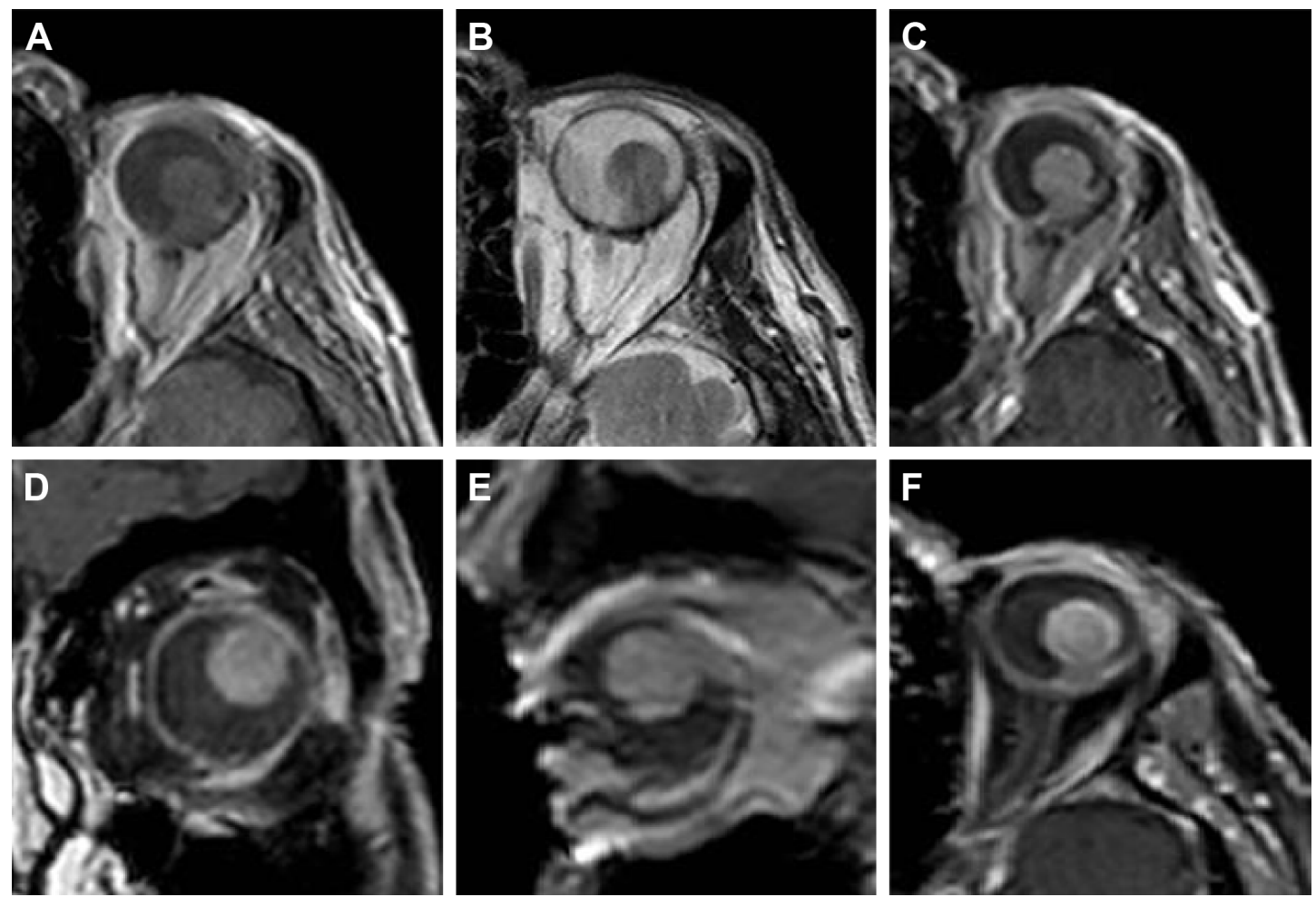

Figure 3 Characteristic appearance of choroidal melanoma on MRI.

Notes: (A) High intensity signal on TI-weighted images, compared with the vitreous body; (B) low intensity signal on T2-weighted images; (C) arterial enhancement on TI-weighted images, after an intravenous bolus administration of paramagnetic contrast agent; (D) appearance on coronal plane; (E) sagittal plane on TI-weighted images, after an intravenous bolus administration of paramagnetic contrast agent; (F) TI-weighted sequence using fat suppression technique, after an intravenous bolus administration of paramagnetic contrast agent.

Abbreviation: MRI, magnetic resonance imaging.

criteria for homogeneity in MRI, we divided the participants into two subgroups: 1) patients with homogenous tumors and 2) patients without homogenous tumors. We compared these two populations through a $t$-test (two-tailed, unequal variances) in terms of the height and base diameter of their tumors.

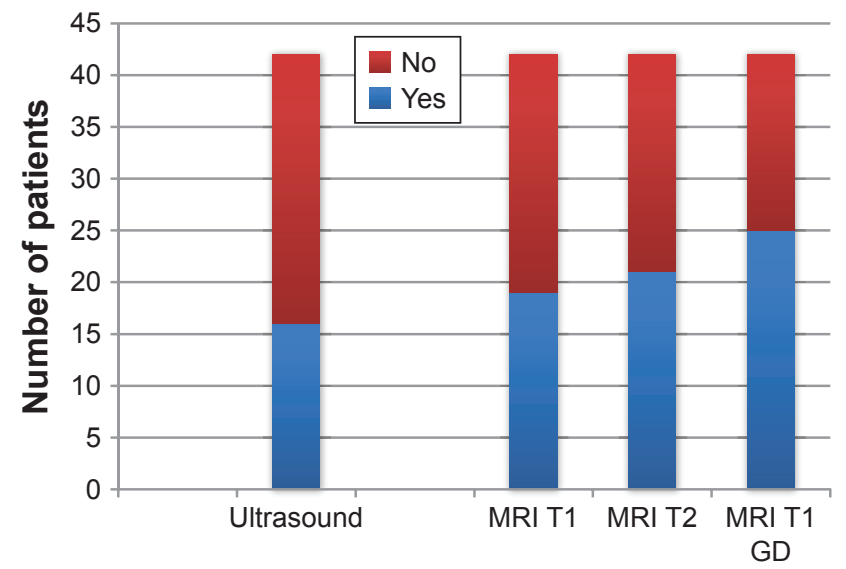

Figure 4 Homogeneity incidence detected by using different methods. Abbreviations: GD, gadolinium; MRI, magnetic resonance imaging.
The patients with homogenous tumors (characterized through T1-WI sequence) presented with a mean height of $6.18 \pm 3.71 \mathrm{~mm}$ and mean base diameter of $10.3 \pm 3.46 \mathrm{~mm}$, and the patients with nonhomogenous tumors presented with a mean height of $12.14 \pm 3.45 \mathrm{~mm}$ and mean base diameter of $13.95 \pm 3.72 \mathrm{~mm}$. By comparing these two populations, a statistically significant difference was found for height, but the correlation was marginally negative for base diameter (height $P=0.0045$; base diameter $P=0.056$ ). Thus, melanomas of greater height were nonhomogeneous, due to increased tissue degeneration and intratumoral bleeding, owing to their increased vascularity.

\section{Correlation of choroidal melanoma's size with tumor's vascularity}

A comparison between the patients with tumors of high vascularity and those with tumors of low vascularity was done in terms of the size of the tumor (ie, height and base diameter). The studied subgroups were compared using a Student's $t$-test for continuous data, regarding the tumor's height and base diameter. 


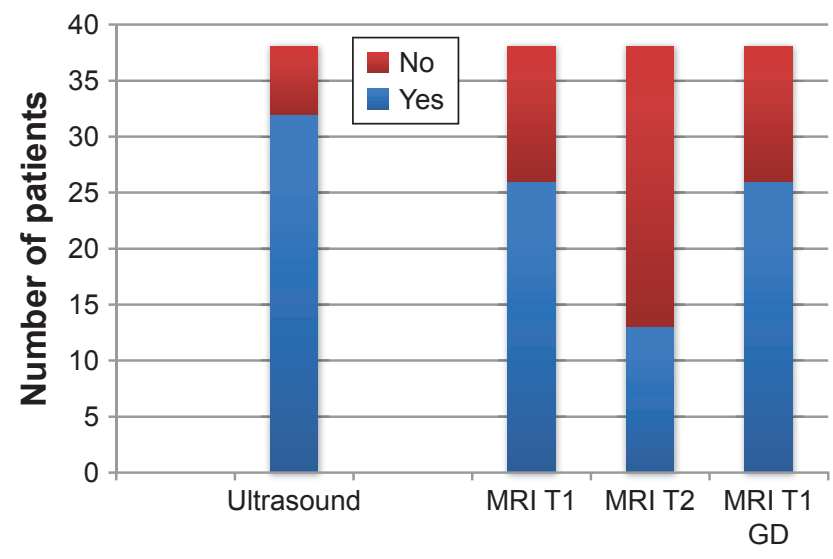

Figure 5 Number of cases where retinal detachment was detected by using different methods.

Abbreviations: GD, gadolinium; MRI, magnetic resonance imaging.

The patients with tumors of high vascularity (characterized through ultrasound scan) presented with a mean height of $10.15 \pm 3.79 \mathrm{~mm}$ and mean base diameter of $13.11 \pm 3.07 \mathrm{~mm}$. In contrast, the patients with tumors of low vascularity presented with a mean height of $2.3 \pm 1.67 \mathrm{~mm}$ and mean base diameter of $7.72 \pm 3.44 \mathrm{~mm}$. By comparing these two populations, a statistically significant difference was found for the height (at the level of 0.001 ) and also for base diameter (at the level of 0.05 ; height $P=0.000638$; base diameter $P=0.019543$ ). Tumor size was statistically significantly correlated with its degree of vascularity, and vascularity was shown to be dependent on one of the two basic measurements of choroidal melanoma's size, which was its height.

\section{Ultrasound scan and MRI sensitivity on RD}

$\mathrm{RD}$ was detected in 38 patients (of the total 42 patients) through direct fundoscopy. Examinations confirmed RD in 32 cases through ultrasound scan, which was proved to be the most sensitive modality. In particular, 26 cases of RD were detected through T1-WI sequence, 13 cases through T2-WI sequence, and 26 cases through T1-WI sequence + contrast (Figures 5-7).

\section{Discussion}

The modalities used in this study to assess the specific choroidal melanoma's imaging characteristics were ultrasound scan and MRI. The ultrasound findings targeted the tumor itself, as well as one of its most frequent complications, namely, RD. Location, shape and size, homogenous structure, and increased vascularity are the most important ultrasound characteristics. Our findings are in accordance with the previous reports on the quantification of characteristics, but nevertheless, it is the first to the best of our knowledge, which attempts to correlate the qualitative characteristics such as homogeneity and vascularity with quantitative parameters such as height and base diameter.

B-mode technique is a two-dimensional method, as the ultrasound beam is being transmitted to a single plane through the orbit. Most important advantages of this modality are lack of radiation, high availability, and high repeatability, while being less expensive than MRI. On the other hand, the experience and expertise of the examiner are important in the ultrasonographic evaluation of orbital pathology. One of the ultrasonography's greatest implementations to the orbit is the diagnosis of malignant melanoma. Success rates in differentiating malignant melanoma from other lesions are high and, according to certain authors, rise $>95 \% .{ }^{16,17}$ Color and power Doppler techniques provide valuable information on tumor vasculature: blood flow rate and velocity.

The MRI is a method of obtaining tomographic images based on the nuclear magnetic resonance phenomenon, without any use of ionizing radiation, and images in this method can be obtained in different ways to bring out the inherent differences of scanned tissues. Signal intensities on T1-and T2-weighted images are related to specific tissue characteristics. The signal intensities, enhancement, and other findings, such as regular borders and homogenous appearance of the normal extraocular muscles and retro bulbar fat, help in identifying normal and abnormal structures. Most important advantages of this modality are high sensitivity, ${ }^{18,19}$ multiplanar imaging, and lack of both radiation and interobserver variation. On the other hand, it is much more expensive than ultrasound technology, it is not highly available, and the examination's duration is much longer; thus, the images are also subject to motion artifacts, for which recent studies suggest dedicated blink/fixation protocols, in order to acquire high-resolution in vivo images with no requirement for postacquisition image registration. ${ }^{20}$ Ocular melanomas containing large amounts of melanin have been shown to have a shorter T2 time and a shorter T1 time than those of other malignant tumors. ${ }^{9,10}$ Thus, these uveal and choroidal tumors appear hyperintense on T1-weighted images and hypointense on T2-weighted images, and this behavior has been attributed to the paramagnetic properties of melanin. Moderate- to high-level enhancement of the tumor is always noted after an IV administration of contrast agent. Magnetic resonance offered the highest resolution and the advantage of multiplanar imaging. In addition, more sequences could be 

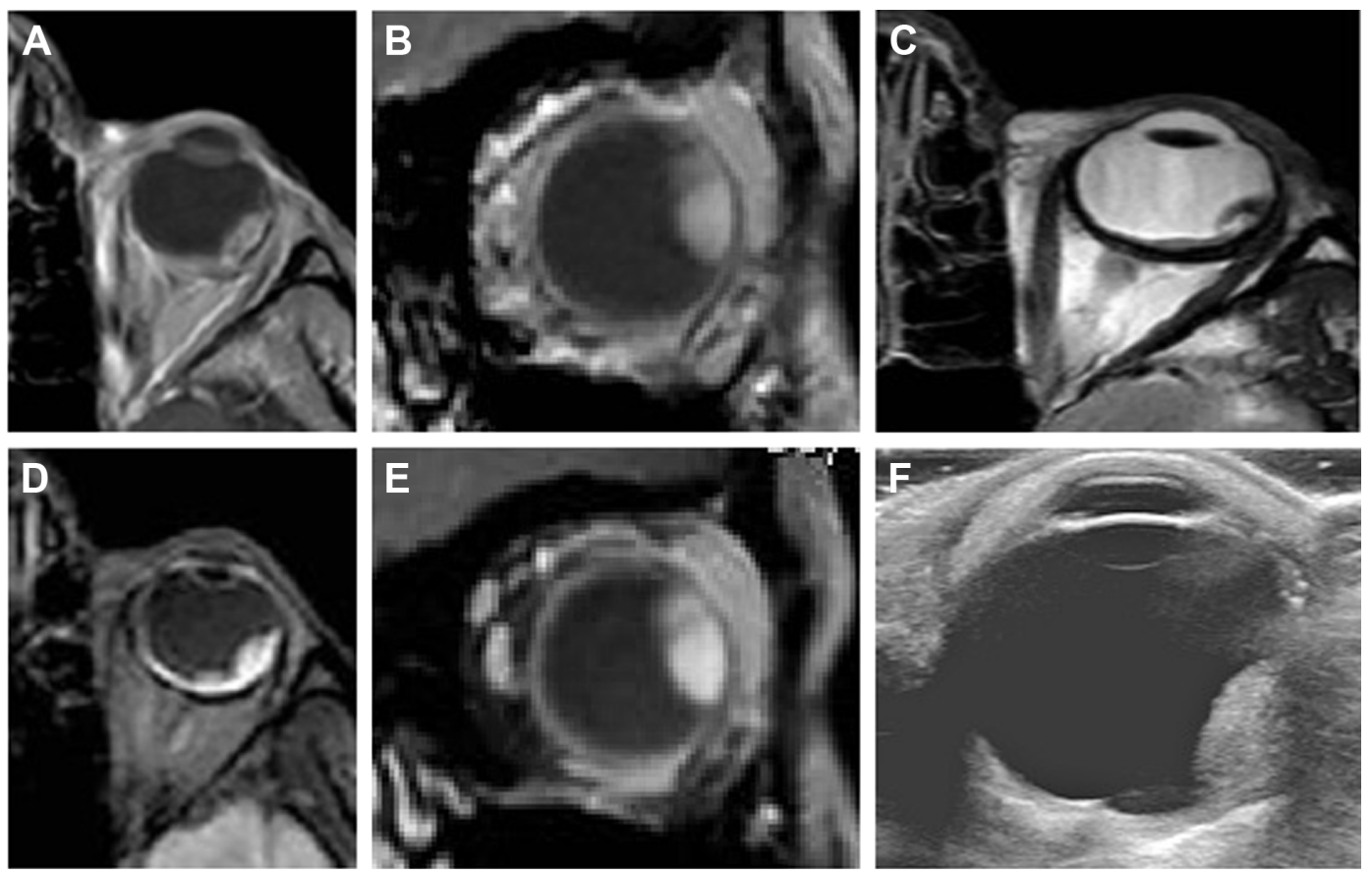

Figure 6 Imaging of choroidal melanoma on MRI and ultrasound scan.

Notes: (A) Tumor showing high intensity signal on TI-weighted images. Its heterogeneous appearance accounts to the melanin distribution pattern. A small retinal detachment may be depicted; (B) TI-weighted image on a coronal plane; (C) heterogeneous appearance and low intensity signal on T2-weighted images for the choroidal melanoma. The retinal detachment is apparent; (D) tumor showing homogeneous arterial enhancement on TI-weighted images with fat suppression, after an intravenous bolus administration of paramagnetic contrast agent. The retinal detachment is shown; (E) TI-weighted image with fat suppression, after an intravenous bolus administration of paramagnetic contrast agent on a sagittal plane; (F) for the same patient, the small retinal detachment is noted on ultrasound scan.

Abbreviation: MRI, magnetic resonance imaging.

selected, adding more information to the study on choroidal melanoma's MRI characteristics.

The parameters studied on MRI were melanoma's location, shape, size, and homogeneity. Mild-to-moderate contrast-enhancing patterns on MRI are key features for the diagnosis of choroidal melanoma and tumor differentiation from other lesions. Our study's results found choroidal melanoma to present homogenous both on ultrasound scan and MRI. This corresponds well to other reports on international bibliography. ${ }^{21-23}$

Choroidal melanoma's size was correlated with the tumor's homogeneity. In particular, its height alone (not the base diameter) was proven to alter homogeneity findings on MRI. Hence, choroidal melanomas of greater height were less homogenous, due to their greater degree of degeneration and greater chance of intratumoral bleeding, owing to their increased vascularity. In addition, choroidal melanoma's size was correlated with its vascularity. Again, the tumor's height was found to be of greater significance, in accordance with other previously published studies, where prognosis worsens when height diameter, extraophthalmic extension, or ciliary body invasion increases. ${ }^{11,12}$ Certain studies note that ultrahigh-field (7 T) ocular MRI can yield a more accurate measurement of the tumor dimensions compared with conventional ultrasound, which can result in significant changes in treatment planning. ${ }^{24}$

Concerning RD imaging, both modalities are reliable. Statistical analysis of our results, though, found ultrasound scan to be more sensitive than MRI in the detection of RD. Contrast enhancement on MRI often facilitates the discrimination of the tumor adjacent to the subretinal fluid (Figure 8). Diffusion-sensitized ms-RARE techniques are reported to acquire high-contrast, high-spatial resolution, distortion-free images at 3.0 and $7.0 \mathrm{~T} .{ }^{25}$

\section{Conclusion}

This study focused on specific choroidal melanoma's imaging characteristics for two different modalities: ultrasound scan and MRI. An attempt was made to correlate a couple of melanoma's quality characteristics, namely, homogeneity and vascularity, with quantified parameters, namely, height and base diameter. Choroidal melanoma was found to be homogenous on both ultrasound scan and MRI. Melanomas of greater height, though, were found to be less homogenous, due to increased degeneration and higher occurrence of intratumoral hemorrhage. In addition, choroidal melanoma's 

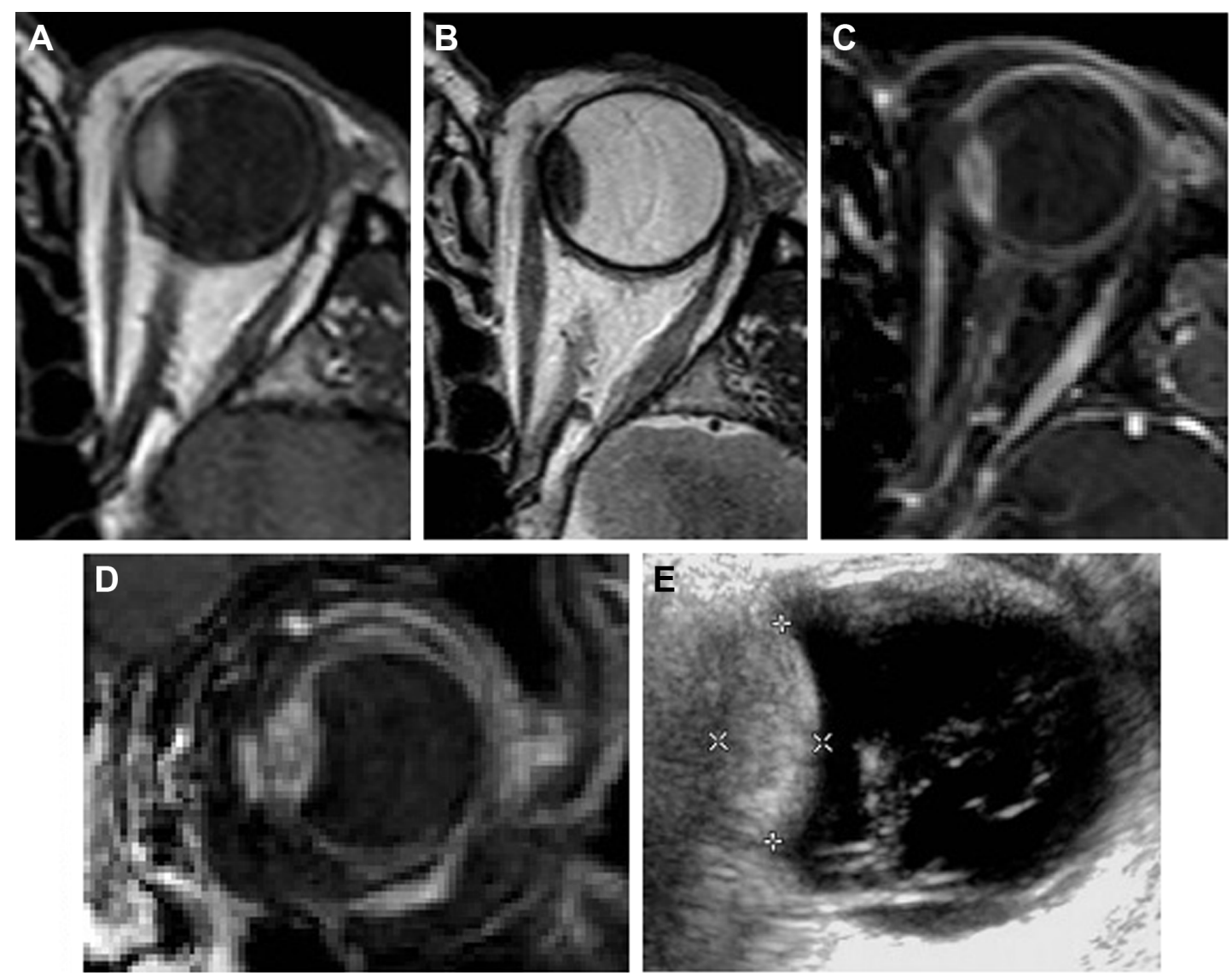

Figure 7 Choroidal melanoma on MRI and ultrasound scan.

Notes: (A) Homogeneous intraocular mass showing high intensity signal on TI-weighted images; (B) on T2-weighted images, it shows low intensity signal; (C) the tumor showing heterogeneous arterial enhancement on TI-weighted images with fat suppression, after an intravenous bolus administration of paramagnetic contrast agent; (D) TI-weighted image with fat suppression, after an intravenous bolus administration of paramagnetic contrast agent on a coronal plane; (E) for the same patient, ultrasound scan revealed a retinal detachment which is undetected by the MRI.

Abbreviation: MRI, magnetic resonance imaging.
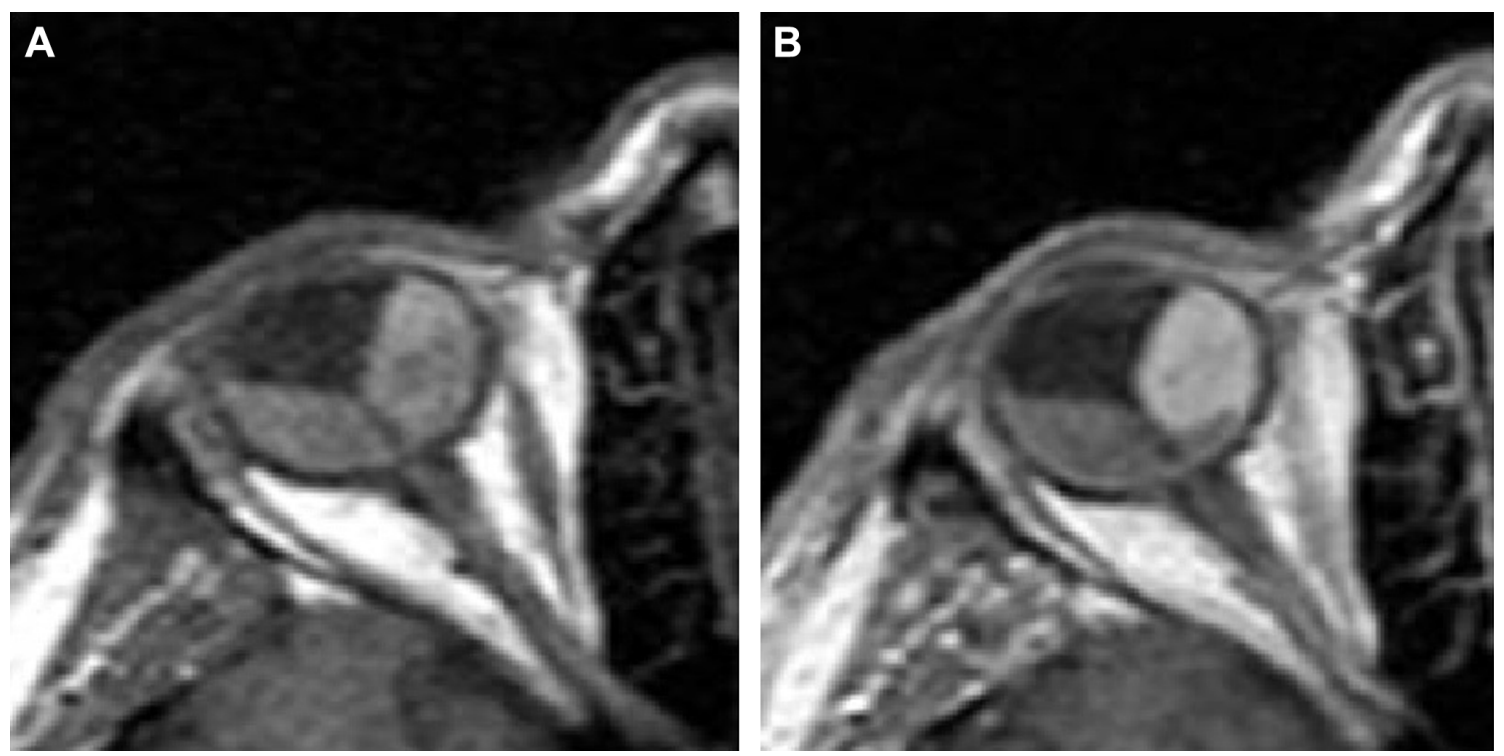

Figure 8 Arterial enhancement of choroidal melanoma on MRI scan enabling imaging differentiation between the tumor and retinal detachment.

Notes: (A) Before an intravenous bolus administration of paramagnetic contrast agent; (B) after an intravenous bolus administration of paramagnetic contrast agent. Abbreviation: MRI, magnetic resonance imaging. 
height was also positively correlated with increased vascularity. For the detection of RD, ultrasound scan was found to be more sensitive than MRI.

\section{Acknowledgments}

This project was part of the first author's (VP) PhD thesis in Aristotle University of Thessaloniki, Greece. Dr Tsaousis received a scholarship (2015) from the Hellenic Society of Intraocular Implants and Refractive Surgery for postgraduate training. The authors thank Dr Anastasia Sarafi for her contribution in data collection.

\section{Disclosure}

The authors have no financial or proprietary interest in any materials or methods described in this study; they report no conflicts of interest in this work.

\section{References}

1. Scotto J, Fraumeni JF Jr, Lee JA. Melanomas of the eye and other noncutaneous sites: epidemiologic aspects. J Natl Cancer Inst. 1976;56: 489-491.

2. Singh AD, Topham A. Incidence of uveal melanoma in the United States: 1973-1997. Ophthalmology. 2003;110:956-961.

3. Shields JS, Shields CL. Posterior uveal melanoma clinical and pathologic features. In: Shields JS, Shields CL, editors. Intraocular Tumours - A Text and Atlas. Philadelphia: W.B. Saunders; 1992:117-136.

4. Shields CL, Pellegrini M, Ferenczy SR, Shields JA. Enhanced depth imaging optical coherence tomography of intraocular tumors: from placid to seasick to rock and rolling topography-the 2013 Francesco Orzalesi Lecture. Retina. 2014;34:1495-1512.

5. Shah SU, Kaliki S, Shields CL, Ferenczy SR, Harmon SA, Shields JA. Enhanced depth imaging optical coherence tomography of choroidal nevus in 104 cases. Ophthalmology. 2012;119:1066-1072.

6. Filloy A, Caminal JM, Arias L, Jordán S, Català J. Swept source optical coherence tomography imaging of a series of choroidal tumours. Can J Ophthalmol. 2015;50:242-248.

7. Yang WL, Wei WB, Li DJ. Quantitative parameter character of choroidal melanoma in contrast-enhanced ultrasound. Chin Med J (Engl). 2012;125:4440-4444

8. Guthoff R, Winkler P, Hebmke K, Berger R. Diagnosis and treatment control of choroidal melanomas: the role of B-scan and Doppler technique. Acta Ophthalmol Suppl. 1992;204:59-61.

9. Gomori JM, Grossman RI, Shields JA, Augsburger JJ, Joseph PM, DeSimeone D. Choroidal melanomas: correlations of NMR spectroscopy and MR imaging. Radiology. 1986;158:443-445.

10. Mafee MF, Peyman GA, Peach JH, Cohen SB, Mitchell MW. Magnetic resonance imaging in the evaluation and differentiation of uveal melanoma. Ophthalmology. 1987;94:341-348.

Clinical Ophthalmology

\section{Publish your work in this journal}

Clinical Ophthalmology is an international, peer-reviewed journal covering all subspecialties within ophthalmology. Key topics include: Optometry; Visual science; Pharmacology and drug therapy in eye diseases; Basic Sciences; Primary and Secondary eye care; Patient Safety and Quality of Care Improvements. This journal is indexed on Submit your manuscript here: http://www.dovepress.com/clinical-ophthalmology-journal
11. Raivio I. Uveal melanoma in Finland: an epidemiological, clinical, histological and prognostic study. Acta Ophthalmol Suppl. 1977; 133:1-64.

12. Augsburger JJ, Gamel JW. Clinical prognostic factors in patients with posterior uveal malignant melanoma. Cancer. 1990;66:1596-1600.

13. Shammas HJ. Atlas of Ophthalmic Ultrasonography and Biometry. The C.V. Mosby; 1982:112-114.

14. Lemke AJ, Hosten N, Bornfeld N, et al. Erscheinungsbild von Aderhautmelanomen in der hochauflösenden 1,5-T-MRT mit einer Oberflächenspule anhand von 200 konsekutiven Patienten. [Appearance of choroidal melanoma on high resolution MRI using $1.5 \mathrm{~T}$ with a dedicated surface coil in 200 consecutive patients]. Rofo. 1998;169:471-478. German.

15. Mafee MF, Peyman GA. Retinal and choroidal detachments: role of magnetic resonance imaging and computed tomography. Radiol Clin North Am. 1987;25:487-507.

16. Coleman DJ, Silverman RH, Rondeau MJ, et al. Ultrasonic tissue characterization of uveal melanoma and prediction of patient survival after enucleation and brachytherapy. Am J Ophthalmol. 1991;112: 682-688.

17. Munk P, Downey D, Nicolle D, Vellet AD, Rankin R, Lin DT. The role of colour flow Doppler ultrasonography in the investigation of disease in the eye and orbit. Can J Ophthalmol. 1993;28:171-176.

18. Chung EM, Specht CS, Schroeder JW. From the archives of the AFIP: pediatric orbit tumors and tumorlike lesions: neuroepithelial lesions of the ocular globe and optic nerve. Radiographics. 2007;27: 1159-1186.

19. Hosten N, Bornfeld N, Wassmuth R, et al. Uveal melanoma: detection of extraocular growth with MR imaging and US. Radiology. 1997;202: $61-67$.

20. Beenakker JW, van Rijn GA, Luyten GP, Webb AG. High-resolution MRI of uveal melanoma using a microcoil phased array at 7 T. NMR Biomed. 2013;26:1864-1869.

21. Mihara F, Gupta KL, Murayama S, Lee N, Bond JB, Haik BG. MR imaging of malignant uveal melanoma: role of pulse sequence and contrast agent. AJNR Am J Neuroradiol. 1991;12:991-996.

22. Lemke AJ, Hosten N, Bornfeld N, et al. Uveal melanoma: correlation of histopathologic and radiologic findings by using thin-section MR imaging with a surface coil. Radiology. 1999;210:775-783.

23. Adam G, Brab M, Bohndorf K, Gunther RW. Gadolinium-DTPAenhanced MRI of intraocular tumors. Magn Reson Imaging. 1990;8: 683-689.

24. Beenakker JW, Ferreira TA, Soemarwoto KP, et al. Clinical evaluation of ultra-high-field MRI for three-dimensional visualisation of tumour size in uveal melanoma patients, with direct relevance to treatment planning. MAGMA. 2016;29:571-577.

25. Paul K, Graessl A, Rieger J, et al. Diffusion-sensitized ophthalmic magnetic resonance imaging free of geometric distortion at 3.0 and 7.0 T: a feasibility study in healthy subjects and patients with intraocular masses. Invest Radiol. 2015;50:309-321.

\section{Dovepress}

PubMed Central and CAS, and is the official journal of The Society of Clinical Ophthalmology (SCO). The manuscript management system is completely online and includes a very quick and fair peer-review system, which is all easy to use. Visit http://www.dovepress.com/ testimonials.php to read real quotes from published authors. 